\title{
Management of Diabetes during Pregnancy
}

\section{PURPOSE}

The purpose of this guideline is to provide guidance on screening for gestational diabetes mellitus (GDM) and the management of pregnancies complicated pre-gestational (PGDM) and GDM in the Sri Lankan setting.

\section{SCREENING}

\subsection{Target groups for screening}

Being South Asian and pregnant places women in Sri Lanka at a higher risk for diabetes during pregnancy. Therefore, universal screening, using a diagnostic test is recommended for all Sri Lankan women.

A. All pregnant women should be screened for diabetes at the first visit unless they are already known to have Diabetes*. This should be performed as early as possible, preferably before 12 weeks, in order to diagnose previously undetected diabetes.

B. Screening using fasting blood glucose, random blood glucose, SOg glucose challenge test, HBA,cor urinalysis for reducing substances is not recommended.

C. Those who are negative for diabetes at the first visit should be screened for GDM again at 24-28 weeks.

D. Women who are known diabetics should not undergo further screening or diagnostic tests. They should be commenced on glycaemic control measures immediately under the supervision of obstetrician or physician.

\begin{tabular}{|c|c|}
\hline \multicolumn{2}{|c|}{$\begin{array}{l}\text { *Diagnostic criteria for pre pregnancy diabetes are anyone } \\
\text { of the following }\end{array}$} \\
\hline FBS & $\geq 126 \mathrm{mg} / \mathrm{dl}$ \\
\hline RBS & $>200 \mathrm{mg} / \mathrm{dl}$ \\
\hline $\mathrm{HbA}_{1 \mathrm{c}}$ & $>6.1 \%$ \\
\hline
\end{tabular}

\subsection{Recommended tests}

A. One stage, non- fasting 7Sg OGCT as described by the Diabetes in Pregnancy Study Group of India (DIPSI) is recommended for screening at the first visit and at 28 weeks. A 2- hour blood glucose of more than $140 \mathrm{mg} / \mathrm{dl}$ confirms gestational diabetes. This is the recommended test for both field and institutional levels.

\section{One stage Non- fasting $75 \mathrm{~g}$ OGCT}

In this method $75 \mathrm{~g}$ oral glucose load is given to the woman irrespective of the fasting status. Therefore a woman could be subjected to a GTT at any time of the day, without having to fast.

A load of 75 of glucose dissolved in $300 \mathrm{ml}$ water is given over 3-5 minutes. The water may be flavoured with lime juice.

The plasma glucose level is measured after a period of two hours.

(The main advantage of this test is that it would be the best way to ensure universal screening. The advantages include reduced cost, the ability to make a diagnosis in one test and the woman not requiring to fast for the test. The test has been validated against the WHO and HAPO criteria and been found to correlate well with them (3),(4). Data also shows that glucose levels are not significantly affected by the fasting status and that the non-fasting glucose level effectively predicts adverse effects for the mother and baby (5),(6).)

B. Three-point oral GTT - In the event of an equivocal screening result or when resources permit, the three point OGTI is recommended. For those who undergo three point OGTI the following cut off should be used for diagnosis.

\section{Three-point oral GTT}

This is probably the most accepted diagnostic test in the world today.

The woman should attend for the test having fasted for eight hours or more, having had a diet unrestricted in carbohydrates.

Blood is first drawn for estimation of fasting plasma glucose.

The woman is then given a solution of $75 \mathrm{G}$ glucose dissolved in $300 \mathrm{ml}$ of water to be taken within 10 minutes. Squeezing a lime into this water will make the solution more palatable without interfering with the result.

Blood is then drawn at 60 and 120 minutes for estimation of plasma glucose.

C. In situations where neither of the above tests is possible, (Inability to tolerate glucose or non availability of facilities) two-stage screening using a 2 hour PPBS is an alternative. The cut off blood glucose value to refer for a OGTT is $\sim 120 \mathrm{mg} / \mathrm{dl}$. 


\section{2 hour Post Prandial Blood Glucose Testing (PPBS)}

Advice the woman to have normal diet.

The time of starting the meal needs to be noted. The meal should be completed within 15 minutes.

The two-hour cut off is calculated from the time of starting the meal.

At the end of two hours blood sample should be tested for blood sugar levels using glucometer or other laboratory method.

\section{MANAGEMENT - WOMEN WITH ESTABLISHED DIABETES}

\subsection{Pre Pregnancy care}

The importance of avoiding unplanned pregnancy is an essential component of diabetes education for women with diabetes.

Women with diabetes who are planning to become pregnant and their families should be offered information on how diabetes affects pregnancy and how pregnancy affects diabetes.

Discuss their plansfor pregnancy and reinforceanappropriate contraceptive method. Any type of contraception can be used except for women BMI $>25 \mathrm{~kg} / \mathrm{m}^{2}$ where DMPA should not be used. Pregnancy is contraindicated if the woman has proliferative retinopathy, stage 2 or above chronic kidney disease or major cardiac disease.

All women with diabetes wishing to conceive MUST be encouraged to seek specialist advice to ensure satisfactory glycaemic control $\left(\mathrm{HbA}_{1 \mathrm{c}}<6.1 \%\right)$ before conception.

Ideally the decision to embark on pregnancy in known diabetics should be decided on based on her $\mathrm{HbA}_{1}$. A value of 6.1 or below would be ideal if safely achievable. Women whose levels are above $10 \%$ should be strongly advised against conception until good glycaemic control is achieved, in view of higher risk of congenital anomalies.

Stress that good planning and control will help to achieve pregnancy outcome to be equivalent to that of a non-diabetic women. They should be informed that establishing good glycaemic control before conception and maintaining this throughout pregnancy will reduce the risk of miscarriage, congenital malformation, still births and neonatal deaths.

Women who are using either metformin or insulin for glycaemic control should be advised that these are safe for use during the peri-conception period and into their pregnancy. Self-testing of blood sugar should be encouraged wherever economically feasible.

Women must be encouraged to achieve a normal weight before becoming pregnant, especially those with a body mass index above $25 \mathrm{~kg} / \mathrm{m}^{\prime}$ - They must receive advice about reducing weight using lifestyle modification.

Known diabetics should be assessed for diabetic nephropathy and retinopathy before and during pregnancy. (see below)

Start Folic acid $\underline{5 \mathrm{mg} \text { daily }}$ when trying to conceive.

\subsection{Antenatal Care}

\section{At the first visit}

- $\quad$ Refer for specialist care immediately once identified. These women are best managed with combined inputs from a physician and an obstetrician.

- Start/ continue Folic acid 5mg daily up-to 12 weeks of gestation. Change to $\mathbf{1} \mathbf{m g}$ daily from 12 weeks onwards.

- Low dose Aspirin (75 mg daily) should be commenced, if there is no contraindication.

- $\quad$ Check $\mathrm{HbA}_{1 \mathrm{c}}$ (ideally $6.1 \%$ or less).

- Dating ultrasound scan using either crown rump length or head circumference is recommended.

- Women with pre-existing diabetes mellitus must be screened for diabetic end-organ damage (retinopathy, nephropathy and cardiovascular disease)

- Retinopathy screening is recommended at least twice during pregnancy (at first contact and at 28 weeks).

- Women with serum creatinine $>120 \sim \mathrm{mol} /$ litre or 24 hour urinary protein excretion exceeding $300 \mathrm{mg}$ must be referred for renal specialist's advice.

- Women with complicated diabetes should be managed at a tertiary care institution by a multidisciplinary team.

\section{Antenatal Appointments}

- These women must be identified as high risk and managed almost entirely by a specialist Obstetrician led team.

- Public Health Midwife should visit such women once in every 2 weeks (refer guideline on domiciliary care for high risk pregnancies).

- $\quad$ Review by the obstetric/diabetic team once every 2 weeks throughout the pregnancy

- Anomaly scans at 18-20 weeks and Obstetric reviews at 22-24, 28, 32 and 36-37 weeks with ultrasound growth assessments.

- If required, antenatal steroids for fetal lung maturity may be used. Women should be admitted to hospital for glycaemic control during therapy since glucose levels rise in response to steroids.

- More attention should be given to the woman with diabetes during antenatal preparation for breast feeding as they need to start and establish breast feeding quickly to prevent hypoglycaemia of newborn.

- Refer to dental surgeon for screening and maintenance of oral hygiene. 


\subsection{Medical nutrition therapy (MNT)}

MNT is the cornerstone of the management of diabetes in pregnancy. Women must be referred to a dieticianj diabetic educator nurse where one is available.

Emphasise the importance of small frequent meals, food with low glycaemic index. Dietary advice should be culture sensitive.

\subsection{Exercise}

Exercise has an insulin-like action and women with GDM and pre-existing diabetes complicating pregnancy. Therefore, diabetic women must be encouraged to engage in regular exercise.

The intensity of exercise would depend on the woman's level of fitness, presence of complications and familiarity with exercise.

Ideally this should be at least 30 minutes per day of an activity, which leaves her slightly breathless.

Women on insulin must be aware of the tendency to hypoglycaemia and dehydration during exercise.

\section{GLYCEAMIC CONTROL AND MONITORING}

\subsection{Glyceamic Control}

\subsubsection{The aim is to achieve optimum glycaemic control throughout the day for the duration of the pregnancy (avoiding hypoglycaemia)}

The target values for glycaemic control are given below:

\begin{tabular}{|l|l|l|}
\hline \multicolumn{3}{|c|}{ Table 2. Target values in glycemic control } \\
\hline & $\begin{array}{l}\text { Fasting and pre- } \\
\text { meal }\end{array}$ & 2 hour post meal \\
\hline Venous plasma & $\begin{array}{l}70-90 \mathrm{mgjdl}(3.9 \\
-5.0 \mathrm{mMol} / \mathrm{L})\end{array}$ & $\begin{array}{l}\text { Below } 120 \mathrm{mgjdl} \\
(6.7 \mathrm{mMol} / \mathrm{L})\end{array}$ \\
\hline Capillary blood & $\begin{array}{l}80-103 \mathrm{mgjdl}(4.4 \\
-5.7 \mathrm{mMol} / \mathrm{L})\end{array}$ & $\begin{array}{l}118 \mathrm{mgjdl} \\
\text { mMol/L) }\end{array}$ \\
\hline
\end{tabular}

Refer the mother to a Diabetic Educator Nursing Officer (DENa) where one is available.

At diagnosis, offer dietj lifestyle advice with a recorded glycaemic assessment within 1-2 weeks.

Majority of these women can achieve optimal glycaemia with modest changes in diet and exercise. Consider insulin and /or metformin treatment if suboptimal glycaemia persists despite diet and exercise modifications. The choice of these treatments will depend on physician and patient preferences.

Ideally the insulin regimen should be adjusted to achieve targets: in most cases with moderate to severe hyperglycaemia three doses of short acting pre prandial insulin combined with a single dose of basal insulin at bed time is required. However, twice-daily dose of pre mixed 30:70 insulin has better patient compliance with adequate control of blood sugar in most cases. If blood sugar is not controlled by this twice-daily regimen, adding metformin or soluble insulin to cover lunch is an alternative.

Use of ACE inhibitors, statins and ARBs are contraindicated during pregnancy

\subsection{Monitoring of glycaemic control}

Self-monitoring of blood glucose (SM BG) with close liaison with the diabetic team is recommended for those who are able to afford a glucometer and test strips. (However, in view of variable quality of glucometers women must be advised to crosscheck the values occasionally with estimations made by a reliable laboratory.

For women who cannot afford the cost of 5MBG, monitoring with regular 6 point blood glucose monitoring should be offered.

The frequency of such monitoring should be decided by the overall glyceamic control, presence or absence of fetal macrosomia and the period of gestation;: with at least four weekly reviews in pregnancy two weekly reviews in late pregnancy.

Schedule ultrasound measurement of AC at 28, 32 and 36 weeks. If $\mathrm{AC}>90$ centile at any stage, consider insulin therapy to target 2 hour PPBS to be less than $100 \mathrm{mg} / \mathrm{dl}$ but avoiding hypoglycaemia.

If crossing centiles or $\mathrm{AC}<10$ centile, do $\mathrm{AFI}$ and request obstetrician review.

Insulin requirements change throughout the pregnancy. If requirements are falling (or maternal

hypoglycaemia occurs frequently) request early obstetrician review for fetal assessment.

$\mathrm{HbA}_{1 \mathrm{c}}<$ is not a reliable indicator of glycaemic control in the second and third trimesters.

\section{DELIVERY AND INTRA NATAL CARE}

\subsection{Timing of delivery}

For women with pre-pregnancy diabetes or who receive insulin therapy, schedule obstetrician review at 36-37 weeks for planning their delivery at 38-39 weeks.

For women on diet control and/or women having optimal glycaemic control and, carrying a normally grown baby, there is insufficient evidence to suggest the best time for delivery.

Diabetes alone is not an indication for a caesarean section.

The obstetrician should make the decision after discussing with the woman.

Delivery should be arranged in the day time, when all supports are more easily available. 


\subsection{Labour care}

Second tier obstetric on-call (SHO/Registrar) should be informed of any woman with diabetes at the onset of labour. He/she should be present for the delivery. It is recommended to involve the medical team in the management of difficult cases.

Inform on-call neonatal team of any planned/ imminent delivery of a diabetic mother.

During labour and birth, capillary blood glucose should be monitored 1-2 hourly in women with diabetes and maintained at between 4 and $7 \mathrm{mmol} /$ litre. (72 - $126 \mathrm{mg}$ / dl). These CBG records should be entered in the partogram.

Hartmann's / normal saline or Insulin-dextrose - potassium (GIK) infusion should be started if the values are lower or higher respectively.

\section{POST NATAL CARE}

\section{1a. Neonatal care}

Handover care of newborn, to neonatal team.

Ensure delivery-to-abdomen and initiate breastfeeding as early as possible (within first $Y$, to 1 hour) unless specific concerns prevent such action.

Take all suitable essential newborn care (ENC) measures to avoid hypothermia.

Blood glucose testing should be carried out routinely in babies of women with diabetes at 2-4 hours after birth. The mother must be informed about this antenatally to prevent unnecessary distress.

Neonatal blood glucose values below $36 \mathrm{mg} / \mathrm{dl}$ (2 $\mathrm{mMol} / \mathrm{L})$ should trigger action.

Blood tests for polycythaemia, hyperbilirubinaemia, hypocalcaemia and hypomagnesaemia should be carried out for babies with clinical signs.

\section{1b. Immediate post partum care}

It is recommended that the mother be tested for random blood glucose within 4 hours of delivery. The decision to manage maternal diabetes with insulin or oral medication should made within the first 48 hours after delivery and prior to discharge from hospital.

If the mother received insulin in the antenatal period, it is recommended that the dose needs adjustments to pre pregnant doses in those with type 2 diabetes mellitus or be maintained on diet alone in those with GDM. This decision should be based on her post partum blood glucose value. If FBG exceed $126 \mathrm{mgjdl}$ or RBS exceeds $200 \mathrm{mgjdl}$, insulin in a lower dose (usually half of the antenatal dose) or metformin would be required. This decision is best left to the managing physician who should be responsible for the woman's long term care.

\subsection{At hospital discharge}

Inform $\mathrm{MOH}$ and area PHM through woman's pregnancy record.

For women with pre- gestational diabetes, prescribe suitable hypoglycaemic agent, restart statins, schedule follow up clinic date at the medical clinic.

For women who developed GDM, give a date or make arrangements to screen for diabetes mellitus at 6 weeks postpartum (see below for details).

Discuss and help to decide on the suitable contraceptive method.

\subsection{Late Postnatal care and follow up}

At 6 -8 weeks postpartum, all women with GDM are screened for diabetes mellitus. The test of screening is ideally the 75g OGTT. FBS is an alternative if resources are limited. Women whose fasting venous plasma glucose is above $100 \mathrm{mgjdl}(5.5 \mathrm{mMol} / \mathrm{L})$ must be referred for further evaluation.

Women who have been diagnosed with GDM and are screen-negative at the 6 week review should receive lifestyle advice and screening for diabetes mellitus annually with at least a FBS. The importance of maintaining a normal $\mathrm{BMI}$ and the contribution of breastfeeding to weight loss must be emphasized.

\section{FAMILY PLANNING}

8.1 All reliable methods of family planning can be used as appropriate for the needs of the individual woman with diabetes.

8.2 For women with BM I $>25 \mathrm{~kg} / \mathrm{m}^{\prime}$, DMPA is best avoided.

8.3 Women with type 2 diabetes should be advised to complete their family within 5-10 years of diagnosis of diabetes in view of possible development of complications.

\section{REFERENCES}

1. NICE, clinical guideline 63 Diabetes in pregnancy: management of diabetes and its complications from pre-conception to the postnatal period. 2008, National Institute for Health and Clinical Excellence.

2. Seshiah V., Das AK.,BalajiV et aI., Gestational Diabetes Mellitus - Guidelines. Journal of the Association of Physicians of India. 2006. 54: 622- 628

3. IADPSGCP, International Association of Diabetes and Pregnancy Study Groups Recommendations on the Diagnosis and Classification of Hyperglycemia in Pregnancy. Diabetes Care, 2010. 33(3): p. 676-682.

4. Kuhl C. Insulin Secretion and insulin resistance inpregnancy and GDM. Implications for diagnosis 
andmanagement. Diabetes 1991;40(December (Suppl. 2)):18-24.

5. Gough WW, Shack MJ, Bennett PH, Burch TA, Miller M.Evaluation of glucose in the Pima Indians by longitudinal studies. Diabetes 1970;19(Suppl. 1):388.

6. Pettitt DJ, Bennett PH, Hanson RL, Narayan KM, KnowlerWC. Comparison of World Health
Organization and National Diabetes Data Group procedures to detect abnormalities of glucose tolerance during pregnancy. Diabetes Care 1994;17(November (11)):1264-8.

7. Haeckel R., Brinck U, Colic D. et aI., Comparability of Blood Glucose Concentrations Measured in Different Sample Systems for Detecting Glucose Intolerance. Clinical Chemistry 2002.48: (6); 936-939 